\title{
Uso de TIC en investigación cualitativa: discusión y tendencias en la literatura ${ }^{1}$
}

\author{
Use of ICT in qualitative research: discussion and trends in literature \\ Utilização das TIC na pesquisa qualitativa: tendências na literatura e \\ discussão
}

\author{
Gloria María Álvarez Cadavid ${ }^{2}$ \\ María Elena Giraldo Ramírez ${ }^{3}$ \\ Clemencia del Pilar Navarro Plazas ${ }^{4}$
}

Recibido: 02.12.2016 - Arbitrado: 20.12.2016 - Aprobado: 26.01.2017

\begin{abstract}
Resumen
Este artículo es una revisión temática sobre la discusión del uso de TIC en la investigación cualitativa, resultado de la primera fase de un trabajo más amplio cuyo objetivo fue caracterizar los usos de TIC en las prácticas investigativas de docentes - investigadores y su relación con los procesos de formación investigativa en la Universidad Pontificia Bolivariana (UPB) de Medellín, Colombia. Inicialmente se hizo una caracterización cuantitativa de una base de 263 artículos. Se utilizaron descriptores en español e inglés y distintas combinaciones de los mismos: qualitative research, e-research, research computing, e-social science, technology, QDA, Caqdas, software computing, ICT use, research process, data analysis, uso de TIC, investigación cualitativa, tecnologías, proceso de investigación, análisis de datos. La captura de los datos se realizó con el apoyo de software para gestión bibliográfica (Zotero y EndNote) y de distintas bases de datos como Ebsco, Science Direct y Jstor. A partir de esta sistematización se seleccionaron 166 artículos en los cuales se analizó el contenido para
\end{abstract}

\footnotetext{
${ }^{1}$ Este artículo hace parte de la investigación "Usos y apropiación de TIC en las prácticas investigativas cualitativas y en los procesos de formación investigativa en educación superior”, realizada por el Grupo de Investigación Educación en Ambientes (EAV), adscrito a la Facultad de Educación de la Universidad Pontificia Bolivariana (Medellín-Colombia). http://eav.upb.edu.co/peav/ . (This research was developed by Education on Virtual Environments Group, adscribed to the Education Faculty of Universidad Pontificia Bolivariana (Medellín-Colombia) http://eav.upb.edu.co/peav/ )

2 Comunicadora Social, con Maestría en Nuevas Tecnologías de la Comunicación y la Educación de la Universidad Nacional Estudios a Distancia (UNED) de Madrid, España y Doctorado en Procesos de Formación en Espacios Virtuales de la Universidad de Salamanca, España (2013). Docente-Investigadora de la Universidad Pontificia Bolivariana, Coordinadora del Grupo de Investigación en Educación en Ambientes Virtuales (EAV) de la Facultad de Educación Universidad Pontificia Bolivariana (Medellín-Colombia), gloria.alvarez@upb.edu.co

3 Comunicadora Social, con Especialización en Comunicación Audiovisual para la Formación de la Universidad Pompeu i Fabra de Barcelona, Maestría y Doctorado en Comunicación Audiovisual de la Universidad Autónoma de Barcelona. Docente-Investigadora de la Universidad Pontificia Bolivariana desde, Integrante del Grupo de Investigación en Educación en Ambientes Virtuales (EAV) de la Facultad de Educación Universidad Pontificia Bolivariana (Medellín-Colombia), maria.giraldo@upb.edu.co

4 Socióloga de la Universidad Nacional de Colombia. Desempeña labores de asesoría, apoyo y capacitación en el uso de CAQDAS (Computer Assisted Qualitative Data Analysis) para diferentes grupos académicos y de investigación. Estudiante y pasante de investigación del programa de Maestría en Educación de la UPB. Directora Técnica para el Área Cualitativa de la empresa SOFTWAREshop.
}

Citación artículo: Álvarez Cadavid, G., Giraldo Ramírez, M., Navarro Plazas, C. (2017). Uso de TIC en investigación cualitativa: discusión y tendencias en la literatura. Revista Katharsis, N 23, enerojunio 2017, pp.218-235, Disponible en http://revistas.iue.edu.co/index.php/katharsis 
establecer las temáticas correspondientes a procesos investigativos y el uso de software asociado a ellas. Así se pudieron confirmar algunas tendencias ya enunciadas en la literatura y en otros casos, observar prácticas emergentes, asuntos de los que se muestra aquí una discusión vigente para entender los procesos de apropiación de TIC en la producción de conocimiento.

Palabras clave: usos y apropiación de TIC, investigación cualitativa, Caqdas, e-research, investigación formativa.

\begin{abstract}
This paper is a thematic review about the discussion on ICT uses in qualitative research, as a result of a much wider project which objective was to characterize ICT uses in teachersresearcher's practices in investigation and their relations with formative research processes in the Universidad Pontificia Bolivariana (UPB) from Medellín, Colombia. Initially, it was made a quantitative characterization from a base of 263 articles. Both Spanish and English descriptors were used and different combinations of those such as qualitative research, eresearch, research computing, e-social science, technology, QDA, Caqdas, software computing, ICT use, research process, data analysis, uso de TIC, investigación cualitativa, tecnologías, proceso de investigación and análisis de datos. The capture of data was made with the support of bibliographic software support (Zotero and EndNote) and different data bases such as Ebsco, Science Direct and Jstor. From this systematization they were selected 166 articles in which it was analyzed the content in order to stablish the correspondent thematic to research processes and the use of software associated to those. Through this, it could be confirmed some of the tendencies already mentioned in the literature and in other cases, to observe emerging practices, subjects which generate a current discussion to understand ICT appropriation processes in the production of knowledge.
\end{abstract}

Key words: use and ICT appropriation, qualitative research, Caqdas, e-research, formative research

\title{
Resumo
}

Este artigo é uma revisão temática acerca da discussão do uso de TIC na pesquisa qualitativa, resultado da primeira fase dum trabalho mais amplo cujo objetivo foi caracterizar os usos de TIC nos estágios de pesquisa dos docentes -pesquisadores e sua relação com os processos de formação pesquisadora na universidade Pontifícia Bolivariana (UPB) de Medellin, Colômbia. Inicialmente se fez uma caraterização quantitativa dum banco de 263 artigos. Utilizaram-se descritores em espanhol, inglês e diferentes combinações dos mesmos: Pesquisa qualitativa e pesquisa, pesquisa computadorizada e ciência social, tecnologia QDA, Caqdas, software computing, uso ICT, processos de pesquisa, análises de dados. A captura dos dados realizou-se com o apoio de software para a gestão bibliográfica (Zotero y Endnote) e de diferentes bancos de dados como Ebsco, Science Direct y Jstor. A partir desta sistematização selecionaram-se 166 artigos nos quais se analisou o conteúdo para estabelecer as temáticas correspondentes aos processos de pesquisa e o usado software associado a elas. Assim puderam-se confirmar algumas tendências já enunciadas na literatura e em outros casos, observar práticas emergentes, questões dos que aqui apresentam-se uma discussão vigente para entender os processos de apropriação de TIC na produção do conhecimento.

Palavras chave: usa e apropriação das TIC, pesquisa qualitativa, Caqdas, e-research, pesquisa formativa

Citación artículo: Álvarez Cadavid, G., Giraldo Ramírez, M., Navarro Plazas, C. (2017). Uso de TIC en investigación cualitativa: discusión y tendencias en la literatura. Revista Katharsis, N 23, enerojunio 2017, pp.218-235, Disponible en http://revistas.iue.edu.co/index.php/katharsis 


\section{Introducción}

El interés por indagar el uso de TIC en la investigación cualitativa hace parte de un propósito de mayor alcance que pretende entender los procesos de apropiación de TIC para la producción de conocimiento; en otras palabras, hablamos de la mediación tecnológica como presencia permanente en la actividad intelectual humana y bajo una relación simbiótica en la que la tecnología permite nuevos usos y dichos usos terminan por ampliar o modificar los campos de estudio. Y es precisamente, en este escenario de la investigación cualitativa donde se podría entender mejor esa relación.

Desde las primeras experiencias de utilización de las Tecnologías de Información y Comunicación (TIC) en la investigación cualitativa a mediados de la década de los ochenta, se ha planteado el debate sobre los pros y los contras del uso de TIC y, particularmente, de los software tipo Caqdas (Computer-assisted qualitative data analysis software) en el análisis de datos cualitativos. Este debate se sitúa, necesariamente, en el plano epistemológico y metodológico, en el siguiente sentido: ¿El uso de TIC plantea sólo una diversificación en el utillaje técnico utilizado en las prácticas de investigación o va más allá y configura un nuevo enfoque teórico y metodológico en los procesos de investigación cualitativa? (GiraldoRamírez, Álvarez-Cadavid \& Navarro, 2016).

De acuerdo con las evidencias presentadas por Cisneros Puebla (2003), se puede afirmar que el uso de software para análisis cualitativo ya configura un campo de conocimiento independiente. Sin embargo, la discusión frente a las repercusiones de la mediación tecnológica están divididas, pues aún se encuentran posiciones muy arraigadas frente a la adopción de innovaciones y frente al reconocimiento en la manera que los campos de conocimiento se están ampliando e incluso desdibujando en sus límites disciplinares.

\section{Metodología}

Los criterios de selección para el corpus de datos fueron: la pertinencia para el tema, la disponibilidad del documento y la revisión inicial del contenido a partir del título, resumen y cuerpo de la publicación. Con esta selección se procedió a etiquetar otros campos requeridos para la caracterización como el idioma, categorías temáticas y herramientas tecnológicas asociadas a fases específicas de la investigación. Tal como se mencionó se validaron descriptores específicos (qualitative research, e-research, research computing, e-social science, technology, Caqdas, software computing...) se utilizaron bases de datos académicas (Ebsco, Science Direct...) y gestores bibliográficos libres y de pago (Zotero y EndNote. Finalmente se hicieron análisis de datos en Nvivo que consistieron tanto en conteos de palabras, como en análisis de títulos y contenido. El periodo de búsquedas comprendió las dos décadas dado que los reportes del uso de software para análisis de datos cualitativos coinciden con este tiempo.

De acuerdo con el hallazgo central en esta primera fase de revisión bibliográfica se puede hablar de una recurrencia o auge en el estudio y publicación de artículos sobre la temática, a partir del 2002, cuando el tema se torna de interés investigativo y, por tanto, comienza a aumentar el número de publicaciones sobre el uso de TIC en la investigación Citación artículo: Álvarez Cadavid, G., Giraldo Ramírez, M., Navarro Plazas, C. (2017). Uso de TIC en investigación cualitativa: discusión y tendencias en la literatura. Revista Katharsis, N 23, enerojunio 2017, pp.218-235, Disponible en http://revistas.iue.edu.co/index.php/katharsis 
cualitativa. De la misma manera, el idioma predominante en los artículos revisados es el inglés con aproximadamente el $60.66 \%$ de ellos; seguido del español con el 36. 07\% y otros idiomas con el 3.28\% (Giraldo-Ramírez, Álvarez-Cadavid \& Navarro, 2016).

En cuanto a las tendencias en los tópicos tratados se identificaron dos aspectos generales: artículos centrados en el uso de herramientas y artículos en donde se discutía el proceso de investigación a partir del uso de las herramientas. Desde la primera perspectiva, se encontraron referentes que reportaban usos emergentes y comparación entre las distintas funcionalidades de las herramientas. Por su parte, la discusión sobre el proceso investigativo se centró, principalmente, en las fases de sistematización y el análisis de datos, seguida por el proceso de recolección de información en donde se hace más evidente la mediación de TIC en las prácticas investigativas.

Los datos anteriores, sirven de marco para iniciar la discusión acerca de los alcances e incidencia de la mediación tecnológica en la transformación de los modos de hacer y de formar en investigación, de los escenarios en los cuales se desarrolla y de las distintas prácticas que, de acuerdo con las áreas de conocimiento, emergen y se consolidan.

\section{Los matices de la discusión sobre el uso de TIC en investigación: prevención y confianza acerca de las transformaciones}

Uno de los mayores interrogantes que se pudo observar en la literatura frente a la mediación de TIC en los procesos investigativos está referido a si se puede atribuir a dicho uso un cambio en la manera cómo se investiga desde el punto de vista epistemológico, metodológico y práctico. Al respecto, Colás \& De Pablos (2012), hablan de una transformación de la cultura científica cualitativa ya que "la incorporación progresiva de Internet y las herramientas digitales, está transformando de forma silenciosa los métodos y procedimientos de investigación más tradicionales" (p. 77). En concordancia con esta posición, Arriazu (2007), realiza un aporte a la sustentación metodológica de la investigación social on line a propósito del uso investigativo del foro de discusión "estrategia comunicativa" que tuvo gran auge en la primera mitad de la década del 2000. Desde allí, el autor retoma la pregunta acerca de si estas herramientas constituyen nuevos medios o nuevas formas de indagación, analiza la importancia de la comprensión de las transformaciones que ha generado el uso de la tecnología en los escenarios cotidianos para argumentar que, aunque la investigación social on line ha tratado de regirse bajo los preceptos metodológicos tradicionales, las limitaciones conceptuales encontradas ponen de manifiesto la necesidad de "[...] una metodología propia y específica que fundamente y dé pleno sentido a la investigación social en red" (p. 5).

Por su parte, Estalella \& Ardévol (2011) analizan el uso de Internet en investigación social, evidenciando el contraste entre las expectativas de transformación asociadas al uso de las tecnologías y el rezago de las ciencias sociales tanto en su incorporación y aceptación como en el desarrollo de un discurso reflexivo sobre la articulación de estas herramientas con la práctica investigativa. Para estos autores, la posición de la investigación social frente a la incorporación de TIC en sus prácticas "[...] puede caracterizarse, con todos los matices y salvedades necesarias, por un rechazo o desinterés que lleva a una incorporación limitada pese a las grandes expectativas que algunos autores y autoras depositan en ellas" (p. 91). Igualmente, encuentran que la literatura referida al uso de TIC en investigación cualitativa

Citación artículo: Álvarez Cadavid, G., Giraldo Ramírez, M., Navarro Plazas, C. (2017). Uso de TIC en investigación cualitativa: discusión y tendencias en la literatura. Revista Katharsis, N 23, enerojunio 2017, pp.218-235, Disponible en http://revistas.iue.edu.co/index.php/katharsis 
"[...] se centra principalmente en celebrar las posibilidades que se abren y discutir aspectos puramente instrumentales de su uso"(p. 90).

Estalella \& Ardévol (2011) coinciden con los hallazgos que casi una década antes señalaran Cisneros Puebla (2003), Valles (2005) y Bourdon (2002) al encontrar que, a pesar de la creciente importancia del uso de TIC en las ciencias sociales, tanto para los procesos investigativos como para su propio objeto de estudio, esto no ha supuesto en todos los casos ni un uso generalizado ni un proceso reflexivo, que permita articular la mediación tecnológica con los fundamentos metodológicos y epistemológicos de la práctica.

Para Cisneros Puebla (2003) la década de los ochenta constituye un hito en la difusión del ACAC (Análisis Cualitativo Asistido por Computadora o Caqdas por sus siglas en inglés) analizando tres tendencias en la actitud de los investigadores frente a su uso: aquellos que defienden las técnicas manuales, los que prefieren opciones intermedias como el uso de software no especializado y, por último, los usuarios de software desarrollado expresamente, para el análisis cualitativo. A pesar de reconocer en el Caqdas un campo prolífico de producción académica ${ }^{5}$, una gran mayoría de los textos en donde se evidencia la discusión sobre su uso se encuentran en idiomas distintos al español, lo que a juicio de este autor, ha marcado para los países hispanoamericanos un desarrollo disparejo desde la periferia, teniendo como consecuencia una llegada tardía tanto de los programas como de las discusiones que acompañaron su incorporación (Cisneros Puebla \& Davison, 2012; Cisneros Puebla et al., 2006; Fielding \& Cisneros, 2009). La ausencia de estas reflexiones puede conducir a equívocos, como la asociación del uso del software con la validez del análisis, en donde Cisneros (2003) afirma que debe llamarse la atención de los investigadores "[...] en torno a que este tipo de herramientas no "aseguran" por sí mismas, ni el rigor, ni la validez, ni la confiabilidad de la investigación” (p. 303).

Por su parte, Bourdon (2002) analiza un ejemplo concreto de aplicación del software QSR NVivo y QSR Merge en el desarrollo de una estrategia colaborativa entre investigadores. Aunque la ventaja más conocida del software para análisis cualitativo es el ahorro de tiempo ya que permite una mejor organización de los documentos y elementos de análisis asociados a unas potentes funcionalidades de exploración y recuperación de información, Bourdon formula la pregunta de si más rápido no quiere decir también, en cierto sentido, diferente. Para este autor, puede que la inmediatez de los procesos no solo incida en la manera de hacer sino que genere un impacto adicional, por ejemplo redestinar recursos humanos, económicos o técnicos para otras tareas de la investigación; además, resalta la importancia de un dominio de los fundamentos teóricos y metodológicos de la investigación que en ningún caso el software podría sustituir.

Varios autores de esta perspectiva crítica coinciden en resaltar como un aspecto positivo el interés creciente de los investigadores por el tema, la tendencia al aumento de casos de uso y las innovaciones surgidas de las necesidades de los investigadores por superar las barreras técnicas asociadas a los procesos de recolección y análisis, lo que, en últimas, ha permitido el desarrollo de otras formas de hacer la práctica investigativa y la contribución de los investigadores cualitativos en la comprensión de terrenos relativamente novedosos, como ha ocurrido con la antropología visual, la etnografía virtual, el análisis de redes sociales, entre

\footnotetext{
${ }^{5}$ Las evidencias de esta afirmación se pueden ver en el artículo ya referido Tendencias en el uso de TIC en investigación cualitativa. Un panorama de Álvarez, Giraldo y Navarro (sf.) y que es el trabajo complementario de este artículo. Citación artículo: Álvarez Cadavid, G., Giraldo Ramírez, M., Navarro Plazas, C. (2017). Uso de TIC en investigación cualitativa: discusión y tendencias en la literatura. Revista Katharsis, N 23, enerojunio 2017, pp.218-235, Disponible en http://revistas.iue.edu.co/index.php/katharsis
} 
otros (Armony, 1997; Arriazu, 2007; Bourdon, 2002; Carvajal, 2001; Cisnero Puebla, 2003; Estalella \& Ardévol, 2011; Pardo et al., 2012).

Cabe reiterar que en la revisión realizada con respecto al uso de TIC en investigación cualitativa se encontró que los debates se concentraban, casi exclusivamente, en la fase del análisis de datos y más específicamente en el uso de Caqdas, corroborando lo anotado por Valles (2001; 2005), Chernobilsky (2006) y Bourdon (2002) con respecto a la poca evidencia de discusiones similares sobre el uso de herramientas en otras fases de la investigación. Por el contrario, el uso de Caqdas parece tocar una fibra muy sensible que tiene que ver no solo con los aspectos tradicionales de análisis manual en la práctica investigativa cualitativa sino también con sus propios fundamentos teóricos y epistemológicos. Para Chernobilsky (2006), mientras que los creadores de los programas estadísticos podían dar por hecho el conocimiento del procedimiento a aplicar por parte del investigador, en los métodos cualitativos no podía partirse de un supuesto semejante, por cuanto este es un trabajo hermenéutico con unas características particulares.

Autores como Gibbs (2013), Kelle (1997), MacMillan (2005), Mac MacMillan \& Koening (2004), Roberts \& Wilson (2002) hacen eco de la discusión metodológica que en los noventa tuvieron Coffey, Holbrook y Atkinson (1996) con Fielding \& Lee (1996), en relación con las posibles transformaciones negativas en las prácticas de investigación que podría acarrear la popularización del uso de los Caqdas. Por ejemplo, la aparición de una tendencia a equiparar el análisis cualitativo con la teoría fundamentada, como uno de los sustentos teóricos más adecuados para los Caqdas. Al respecto, Kelle (1997) discute lo frágil de esa relación ya que, por una parte, no todo el software cualitativo toma como referente la teoría fundamentada y, por otra, la sola mención de la teoría fundamentada seduce, sobre todo, a los investigadores más novatos en busca de una enunciación más simple de los pasos a seguir para la realización de un análisis cualitativo, siendo esta una decisión más práctica que metodológica. Por su parte, Amezcua \& Gálvez $(2002)^{6}$ reconocen que desde que se generalizó el uso de los Caqdas se evidencia una "[...] tendencia creciente a suplantar la identificación del método utilizado (análisis de contenido, de discurso, etnometodología, etc.) por la denominación del software que se utilizó para el análisis asistido por ordenador [...]”(p. 242).

Para Valles (2005) las posiciones divergentes con respecto a los Caqdas podrían ser una oportunidad importante para discutir acerca de los desafíos y las potencialidades del uso de estas herramientas en investigación cualitativa. Sin embargo, cabe resaltar el carácter social de los procesos de informatización en donde, efectivamente, pueden existir motivos ajenos a las fundamentaciones teóricas y metodológicas, motivos atribuidos a las personas y no a las herramientas. En este sentido, de las situaciones particulares de la mediación tecnológica derivarían distintos tipos de usos y de usuarios, de acuerdo con los niveles de apropiación, tanto del componente técnico como del saber referido al análisis cualitativo, lo que crea la necesidad de promover una conciencia epistemológica con respecto a los usos de TIC. Bourdon (2002), compara esta discusión con la desconfianza generalizada al pasar de la máquina de escribir al procesador de texto, y el proceso gradual de aceptación de esta nueva tecnología para las tareas de oficina. De forma similar, la incorporación del software para el análisis cualitativo de datos debe pasar por un proceso de aceptación y comprensión

\footnotetext{
${ }^{6}$ Manuel Amezcua y Alberto Gálvez (2002) plantean esto, basados en su experiencia como miembros de la redacción de una revista con secciones especializadas en investigación cualitativa.

Citación artículo: Álvarez Cadavid, G., Giraldo Ramírez, M., Navarro Plazas, C. (2017). Uso de TIC en investigación cualitativa: discusión y tendencias en la literatura. Revista Katharsis, N 23, enerojunio 2017, pp.218-235, Disponible en http://revistas.iue.edu.co/index.php/katharsis
} 
para entrar en el terreno de la familiaridad y la confianza, dimensionando su verdadero papel en los procesos de investigación para evitar tanto la fetichización de la herramienta, como las acusaciones de amenazar la pureza metodológica de las investigaciones (Campos \& Godoy, 2008; Carvajal, 2002; Cisneros Puebla, 2003; Valles, 2001, 2005).

También acompañan esta discusión críticas y prevenciones derivadas de las prácticas investigativas individuales, es decir, que se desprenden de la propia experiencia y trayectoria de los investigadores, pues el factor biográfico es clave a la hora de analizar esta temática. Así, se pueden mencionar temores iniciales hacia el uso de software por el distanciamiento que se puede dar entre el dato y el investigador, con los consecuentes análisis desde la superficie (Weitzman \& Miles, 1995 citado en Lee \& Esterhuizen, 2000). Del mismo modo y asociado a la experiencia personal se hace una crítica al tiempo que se demanda del investigador el manejo de algunos programas, quien para incorporarlos y apropiarlos en su práctica deberá modificar sus rutinas de trabajo, lo cual en muchos casos no resulta sencillo. Sin embargo, y, en contraste con lo anterior, tampoco se encontraron en este recorrido investigaciones que presentaran evidencias de que un manejo manual de los datos fuera garantía de una relación íntima con los mismos; aún más, se piensa, por el contrario, que el manejo de información abundante podría resultar abrumadora para los investigadores y que se desperdiciaría el potencial que puede haber en los datos, dadas las limitaciones humanas de procesamiento y organización de información.

\section{Tendencias que emergen: hacia una clasificación de los usos de TIC en investigación cualitativa}

A partir de las diferentes evidencias encontradas sobre usos de TIC en las prácticas investigativas cualitativas y de las discusiones que acompañan estos usos, se realiza a continuación una ampliación de los elementos que, de acuerdo con esta revisión, emergen con mayor fuerza en la literatura revisada.

La multimodalidad de los datos y su tratamiento

Uno de los escenarios de la mediación tecnológica en los que más se han reportado transformaciones es en el campo de los datos visuales. Si bien estas estrategias podrían ser comunes en áreas como el diseño, la ingeniería, el cine y la televisión, entre otros, en el campo particular de las ciencias sociales se puede considerar como una tendencia de surgimiento reciente en donde los formatos de audio y video son cada vez más frecuentes en las investigaciones, en especial en el horizonte de la sociología y la antropología visual. Al respecto, Austin \& Hickey (2009) reflexionan acerca del aporte de las técnicas visuales para la ampliación de las posibilidades de investigación en el área social y la construcción de reportes y experiencias más auténticas e incluyentes. Para Parmeggiani (2009), las TIC están cambiando nuestra manera de trabajar con datos visuales y, para dar cuenta de ello, describe estos cambios mediante cuatro procesos básicos que se han visto potenciados con el uso de dispositivos tecnológicos: archivar, modificar, desplegar y analizar información visual.

Con respecto al primer proceso, archivar, Parmeggiani (2009) destaca la importancia de la organización de imágenes debido a que estas requieren ser clasificadas de acuerdo con atributos que le permitan al investigador diferenciarlas, referenciarlas y localizarlas rápidamente. Para este autor, el formato Html (HyperTex Markup Language) facilita esta tarea debido a que genera un link directo a la imagen asociado a los atributos mencionados. Igualmente, se destacan funciones para almacenamiento de metadatos vinculados a los Citación artículo: Álvarez Cadavid, G., Giraldo Ramírez, M., Navarro Plazas, C. (2017). Uso de TIC en investigación cualitativa: discusión y tendencias en la literatura. Revista Katharsis, N 23, enerojunio 2017, pp.218-235, Disponible en http://revistas.iue.edu.co/index.php/katharsis 
dispositivos como los datos de georeferenciación que permiten reconocer la ubicación exacta de la captura en el mismo instante en que se realiza, aspecto que ha sido de gran utilidad para la localización geográfica de los fenómenos estudiados. Del mismo modo, de acuerdo con la experiencia de las autoras, los gestores bibliográficos podrían constituir un apoyo en este sentido, ya que algunos de ellos permiten incluir archivos multimediales vinculados a datos de referencias que facilitan el archivo de toda la información bibliográfica dentro de una investigación.

Otras posibilidades que se abren para la investigación desde el campo de los datos visuales están relacionadas con los análisis de comportamiento de usuarios por medio de captura de vídeo e imagen desde la pantalla de la computadora. Si bien esta estrategia estaba más relacionada con análisis de usabilidad en las áreas de ingeniería y desarrollo web, ha ido permeando el campo de las ciencias sociales en temas como educación y análisis de procesos de socialización y comunicación mediados por el uso de la tecnología (López, Valdivia \& Fernández, 2016). Para esto se hace uso de software especializado como CamStudio, Camtasia, Screen recorder entre otros, que permiten, por ejemplo, registrar información en video sobre la trayectoria de navegación en internet o el uso de un software y los procedimientos que realiza una persona dentro de un determinado proceso.

Con respecto al segundo aspecto mencionado por Parmeggiani (2009), la modificación de archivos, el autor hace referencia a todas aquellas acciones que se realizan para el mejoramiento de los archivos gráficos, por ejemplo, cuando tienen una resolución deficiente o se quieren unificar en un formato determinado. En el caso de los recursos de audio y vídeo, Bartolomé (2008) describe diferentes posibilidades de tratamiento de información multimedial con el apoyo de nuevas tecnologías y genera unas reflexiones iniciales acerca del papel que puede cumplir el video digital para la investigación en educación, ya que supone nuevas posibilidades en la codificación y construcción de mensajes así como la generación de documentos audiovisuales interactivos.

Frente al tercer aspecto enunciado por Parmeggiani (2009), desplegar, se hace referencia a la necesidad del investigador de poder observar, contrastar y relacionar la información visual de la que dispone para el análisis. En la investigación reportada por este autor, es precisamente la contrastación de fotos tomadas en distintos momentos — diacronía - el aspecto clave para establecer cambios o similitudes en las comunidades observadas. En este sentido, se dispone hoy de herramientas para realizar anotaciones directamente sobre las imágenes o realizar mapas mentales a partir de las mismas, como el programa Virtual Understanding Environment (VUE) (http://vue.tufts.edu).

En el cuarto aspecto mencionado por Parmeggiani (2009), analizar información visual, se hace referencia al proceso de segmentación en categorías de análisis (codificación), así como a la realización de análisis extenso a partir de la vinculación de segmentos de información con memos y anotaciones realizadas por el investigador. Para el caso de los formatos de audio, video, imagen y documentos escaneados se cuenta hoy con programas que permiten señalar y etiquetar fragmentos mediante el uso de códigos. En el caso de la imagen el software QDA Miner permite este proceso y en el caso del audio y del video programas como Transana o Transcriber. Software con robustas aplicaciones para el análisis de datos cualitativos como Atlas.ti y NVivo han incorporado igualmente funciones para trabajar directamente sobre recursos de audio, imagen y video.

Lo anteriormente señalado por Parmeggiani (2009), Brown (2002) lo asocia a la convergencia digital, de la cual afirma ha redefinido la manera en que se colabora y se Citación artículo: Álvarez Cadavid, G., Giraldo Ramírez, M., Navarro Plazas, C. (2017). Uso de TIC en investigación cualitativa: discusión y tendencias en la literatura. Revista Katharsis, N 23, enerojunio 2017, pp.218-235, Disponible en http://revistas.iue.edu.co/index.php/katharsis 
comparte información, dado que hoy estamos en presencia de otras formas de interacción y ello debe motivar la creatividad a la hora de investigar. Pese a lo anterior, en donde se observa un uso creciente de material multimedial, investigaciones como la de Meyer \& Dutton (2009) indagaron por el uso específico del análisis de video mediante software especializado y encontraron que apenas el $4 \%$ de los investigadores consultados lo utilizaba.

Prácticas que devienen en estrategias metodológicas: entre lo que emerge, se renueva o cobra auge

De acuerdo con el análisis realizado de la literatura se pudieron identificar algunas experiencias que se constituyen en estrategias metodológicas, la mayoría de ellas si bien no son nuevas sí se han redimensionado y adquirido un nuevo interés dentro de la comunidad investigativa.

- El Shooting Scripts: esta es una opción metodológica retomada por Parmeggiani (2009) del autor Charles Suchar (1997, citado por Parmeggiani). Esta estrategia da cuenta de una técnica basada en el método de comparación constante presente en la teoría fundamentada que consiste en tomar imágenes con aspectos visuales relevantes y luego hacer una lluvia de preguntas sobre estas, evitando conceptos preexistentes para, a partir de allí, planear la próxima toma de fotos en campo. Lo que resulta aquí más revelador es el uso de datos gráficos como fuente principal de los datos.

- Integración de datos en métodos mixtos: integrar datos textuales y numéricos no sería posible sin los desarrollos del software que hoy permiten importar, con relativa facilidad, datos de diferente naturaleza para su análisis de forma simultánea en el mismo software, factor que ha promovido la exploración y uso de dichas funcionalidades para enriquecer y agilizar las conexiones entre los horizontes cualitativo y cuantitativo.

- Photo-screen elicitation (foto- provocación): si bien es una estrategia metodológica que está documentada desde $1957^{7}$, ésta se renueva con el uso de TIC pues durante este primer proceso de indagación se encontraron en el portal Sage ResearchMethods (de la editorial Sage y especializado en metodología de investigación) en el periodo del 2010 a 2014 aproximadamente 55 registros referidos al tema. La photo provocación es una actividad que se basa en la visualización de imágenes y/o video como material para provocar o involucrar a los sujetos investigados de una manera distinta, ya que se hace con ellos una visualización de imágenes, generalmente durante las entrevistas para lograr una mejor relación entre investigador e investigado (Hill, 2014; Tonge et al., 2013; Wells, Ritchie \& Macpherson, 2013; Fellinghauer et al., 2011; Corredor-Álvarez \& Íñiguez-Rueda, 2016).

Comunicación y representación de datos

En este aspecto lo que más se destaca en la literatura revisada son las posibilidades que brinda el hipertexto como estructura narrativa para la presentación de informes y resultados, y todo lo que tiene que ver con la hipermedialidad en la que se integran los distintos formatos.

\footnotetext{
7 De acuerdo con el portal Sage Reseach Method (http://srmo.sagepub.com/view/sage-hdbk-visual-researchmethods/n11.xml) la Photo-elicitation fue descrita por primera vez por John COLLIER, Jr (1957) y luego fue descrita con más detalle en el libro: Visual Anthropology: Photography as a Research Method (COLLIER and COLLIER, 1986, p. 99125). Sobre este tema también se pueden ver el trabajo de Felipe Corredor Álvarez y Lupicinio Iñiguez Rueda (2016) Citación artículo: Álvarez Cadavid, G., Giraldo Ramírez, M., Navarro Plazas, C. (2017). Uso de TIC en investigación cualitativa: discusión y tendencias en la literatura. Revista Katharsis, N 23, enerojunio 2017, pp.218-235, Disponible en http://revistas.iue.edu.co/index.php/katharsis
} 
Al respecto Brown (2002) destaca las ventajas de los multimedia para la presentación del contexto y las múltiples interpretaciones que pueden tener los datos; pero llama la atención sobre las consideraciones que se deben tener frente al uso del hipertexto porque el que el autor no tiene control sobre la navegación y si no está bien planeada (en concordancia con los hallazgos) puede resultar confusa y difícil de navegar ${ }^{8}$.

En la misma dirección, autores como Lee \& Esterhuizen (2000) advierten que el trabajo hipertextual puede ser un proceso lento o incluso confuso porque se pueden unir muchos objetos de manera compleja y ello implicaría una alta demanda cognitiva, en algunos casos innecesaria, para un lector.

Lo que sí resulta evidente hoy son las facilidades para hacer esquemas categoriales y relaciones semánticas que permiten visualizar los resultados, es decir se tiene una gama de posibilidades para explorar la potencia de la graficación (Laukkanen, 2012; O'brien et al, 2012) no solo para la etapa de análisis de datos sino para la comunicación de resultados cualitativos, aspecto en el que la graficación cuantitativa de datos lleva un largo camino.

Lo que dejan ver estas evidencias es que, efectivamente, estamos en presencia de tecnologías y prácticas emergentes ${ }^{9}$ para la investigación que están incidiendo en las formas cómo se enfrenta y se realiza la investigación. En este sentido, se puede afirmar que se han ampliado, modificado, potenciado y creado nuevos procesos y escenarios para la investigación, a partir de la mediación de TIC, los cuales se pueden sintetizar como sigue:

- Potenciación de los procesos de validación: en este sentido se encontraron diferentes prácticas que se han visto mejoradas por los facilidades que aportan las TIC:

- Los resultados preliminares de una investigación se publican (un borrador) y se dan a conocer en grupos o redes académicas especializadas en distintos lugares, con el fin de recibir una retroalimentación y mejorar esos primeros análisis.

- Los datos empíricos digitalizados se comparten con otros investigadores (del mismo equipo del proyecto) para que cada uno haga un análisis que luego se contrasta desde las coincidencias y diferencias.

- Los análisis realizados por un investigador o grupo se comparten con pares (investigadores de otros grupos) para que puedan validarlos.

- Datos empíricos que se comparten y son re-utilizados, ya sea para ampliar el estudio a partir de análisis secundarios o para realizar réplicas del estudio en otros escenarios. $^{10}$

- Ampliación de escenarios: este ámbito ha tomado forma de la mano del crecimiento de internet, es decir, de la dinámica misma de la red. Un ejemplo de ello lo configuran los

\footnotetext{
8 Un trabajo interesante en este sentido por el tema y por la forma de presentarse se puede ver en: http://www.exploringthehyper.net/omeka/exhibits/show/about/how-to-use

${ }^{9}$ Por tecnologías emergentes se entiende todas aquellas herramientas, aplicaciones o dispositivos tecnológicos que han sido poco difundidas y por tanto, están aún en periodo de adopción en muchos campos.

${ }^{10}$ En 2005 Forum: Qualitative Research (FQS), dedica su número 6(1) a revisitar el tema del análisis secundario de datos, pero esta vez con las posibilidades que brindan los nuevos sistemas y métodos técnicos de archivo de datos a la re-utilización y al análisis secundario. También se encuentran discusiones acerca de los peligros que puede representar la re-utilización y el análisis secundario de datos, por ejemplo, por la pérdida del contexto en la información.

Citación artículo: Álvarez Cadavid, G., Giraldo Ramírez, M., Navarro Plazas, C. (2017). Uso de TIC en investigación cualitativa: discusión y tendencias en la literatura. Revista Katharsis, N 23, enerojunio 2017, pp.218-235, Disponible en http://revistas.iue.edu.co/index.php/katharsis
} 
estudios sobre las redes sociales y las diversas formas de socialización, comunicación interpersonal, configuración de ciudadanía en el ciberespacio, entre otros. De manera, concreta, por ejemplo, Facebook y Twitter se han convertido en uno los escenarios más nombrados y estudiados en los últimos cinco años (Pérez Da Silva et al, 2013; Giglietto. Rossi \& Bennato, 2012; Aparaschivei, 2011) ${ }^{11}$.

- Incremento de diseños metodológicos mixtos: las posibilidades tecnológicas de los software han facilitado el trabajo con datos cuantitativos y cualitativos, con lo cual se pueden manipular datos textuales y estadísticos haciendo cada vez menor la brecha entre ambos paradigmas y por el contrario beneficiándose de su complementariedad (Crowley, Harré \& Tagg, 2002).

- Facilitación de la identificación de relaciones entre segmentos de datos: funciones presentes en software como Atlas-ti y Nvivo permiten lo que se conoce como la hipervinculación de segmentos, es decir la construcción de relaciones contextuales. Esto se puede entender mejor en el caso en que una codificación de datos se dificulta porque, por ejemplo, hace parte de una narración y al segmentarla se pierden las relaciones con el contexto. Esta posibilidad ha sido destacada desde hace varios años por Weaver \& Atkinson (1994 citados en Lee \& Esterhuizen, 2000) y en ella resaltan su similitud con análisis heurísticos, aspecto muy congruente con la investigación cualitativa.

- Trabajo simultáneo con varios tipos de formatos de datos: hay software que permite hoy segmentar, por ejemplo, los archivos de sonido o texto por tiempos o por hablantes y visualizar a la vez audio y texto en una misma pantalla. Autores como Alcock \& Iphofen, (2007) señalan que esta posibilidad le permite a un investigador tener a mano el audio original por si requiere ver otra información de contexto.

- Optimización de los procesos de transcripción: en relación con el ítem anterior, las TIC permiten hacer de dicho proceso una actividad más ágil, tal como lo reportan también Alcock \& Iphofen (2007), quienes hablan de las posibilidades de un software como el Transcriber que es compatible con Nvivo y que permiten hacer varias actividades al tiempo como por ejemplo transcripciones selectivas sin perder el contexto de lo que se selecciona. Además un investigador puede tener a mano el audio original, dado el caso de que la transcripción no sea muy confiable o, como ya se dijo, de que quiera ver otra información de contexto.

La transcripción, contrario a lo que se pensaría, es un tema importante porque al ser un trabajo dispendioso, realizado generalmente por terceros exige personas con experiencia que garanticen la fiabilidad del dato, incluso Alcock \& Iphofen afirman que la transcripción luego de una entrevista, de alguna manera, ya es una interpretación y, por ello, se constituye en un punto sensible en la discusión que no se puede reducir al uso de un software. Este tema deja abierta la discusión sobre si hoy las transcripciones textuales (Verbatim) son absolutamente necesarias (Cope, 2014).

\footnotetext{
${ }^{11}$ Un ejercicio general de búsqueda en una base de datos multidisciplinar como Ebsco con los descriptores "Facebook y Twitter" arroja como resultado aproximadamente 11.400 registros relacionados con estos dos escenarios de socialización. Citación artículo: Álvarez Cadavid, G., Giraldo Ramírez, M., Navarro Plazas, C. (2017). Uso de TIC en investigación cualitativa: discusión y tendencias en la literatura. Revista Katharsis, N 23, enerojunio 2017, pp.218-235, Disponible en http://revistas.iue.edu.co/index.php/katharsis
} 
- Incremento del uso de datos visuales: aquí se hace referencia a investigaciones que se basan en datos visuales que tradicionalmente se han obtenido mediante registro fotográfico pero que hoy implican otro tipo de procesamiento. Tal vez uno de los trabajos más completos revisados para este texto fue el de Parmeggiani (2009), ya mencionado en extenso, quien afirma que las TIC están cambiando la manera en que tomamos, vemos, almacenamos, analizamos y mostramos imágenes.

- Consolidación de nuevas prácticas metodológicas: esto se refiere a lo que se denomina como métodos online de investigación o $e$-researh ${ }^{12}$ en donde se pueden encontrar métodos tradicionales pero renovados tales como la etnografía virtual ${ }^{13}$ que hace uso de diferentes técnicas mediadas por TIC, pero, además, tiene como objeto de estudio lo que acontece en las comunidades virtuales desde el punto de vista sociocultural. También se pueden mencionar el hyperlinksresearch (web link analysis) o el hiperlink network analysis en donde se combina todo lo que corresponde desde el ámbito cuantitativo a la minería de datos (data mining) y en general los estudios de vínculos en redes conocidos como análisis de redes sociales (ARS) en los cuales se estudian los enlaces o rutas de navegación de los usuarios en internet (Liu, Shih \& Tsai, 2011; De Bakker \& Hellsten, 2013; Lang, Gouveia \& Leta, 2013). Igualmente, se encuentran estrategias metodológicas específicas soportadas por software para análisis de conversaciones cotidianas en organizaciones sociales (Zelger y Oberprantacher, 2002).

- Auge de la cultura de la abierta para la distribución de los datos: Las prácticas de la cultura digital devienen cada vez en el Open Access, Open Source, Open data, Open Science, Open University. Significa el paso de la cultura de la concentración a la cultura distribuida, de la individualidad a la colaboración ampliada e híbrida, esto es, no necesariamente circunscrita a lo académico. En otras palabras es lo que Tapscott \& Williams llamaron wikificación (2007, que aplicado al ámbito de la investigación se convierte para Kenneth S. Kosik en wikificación del conocimiento (2008), redes de colaboración a gran escala, sobre todos de novatos anónimos, a los cuales llama Nigel Fielding (2012) "investigadores ciudadanos", es decir, personas sin formación académica tradicional, pero a los cuales la práctica social con fines no académicos los ha llevado a la investigación como alternativa de comprensión y conocimiento de su realidad particular, para fines concretos, propios de su comunidad, y a quienes poco les interesa una legitimación en los términos de los estándares de la ciencia y se concentran en procesos de innovación abierta.

\footnotetext{
${ }^{12}$ En el 2006 Paul Wouters y Anne Beaulieu proponen el concepto de e-research para referirse a la incorporación progresiva de la TIC a la producción del conocimiento de las ciencias sociales. Sobre el concepto Estalella (2011) dice que Wouters y Beaulieu no hacen referencia a la "e" en alusión a electronic sino a "enhaced" para expresar que lo que se introducen son mejoras en el proceso.

${ }^{13}$ Para ampliar este tema de otras posibilidades de estudios de la red y las prácticas investigativas que se han derivado de esto ver: Álvarez Cadavid (2009). Etnografía virtual: exploración de una opción metodológica para la investigación en entornos virtuales de aprendizaje. (Artículo de reflexión no derivado de investigación.) Revista Q, 3 (6), 12, enero-junio. Disponible en: http://revistaq.upb.edu.co
}

Citación artículo: Álvarez Cadavid, G., Giraldo Ramírez, M., Navarro Plazas, C. (2017). Uso de TIC en investigación cualitativa: discusión y tendencias en la literatura. Revista Katharsis, N 23, enerojunio 2017, pp.218-235, Disponible en http://revistas.iue.edu.co/index.php/katharsis 


\section{Conclusiones}

El ejercicio de revisión y análisis de la literatura realizado en este artículo permitió constatar el auge de prácticas que lentamente se van consolidando como tendencias metodológicas, tal es el caso de la etnografía virtual y la antropología visual, en cuyos campos se han ampliado y redefinido los escenarios investigativos, por ejemplo, la socialización y la interacción se ha desplazado a espacios como los foros, blog y redes sociales que, en general, han complejizado la comunicación humana. Del mismo modo, se observa la ampliación en el acceso, tanto a dispositivos, como a aplicaciones para el registro y captura de datos en audio, imagen y video que entran a enriquecer los formatos textuales de los corpus de análisis $\mathrm{y}$, al tiempo, aumentan la disponibilidad de gran cantidad de datos que requieren mayor capacidad de procesamiento.

Este panorama resulta de particular interés en el contexto actual de la producción de conocimiento en el ámbito académico donde aumenta la presión por la publicación de resultados en tiempos cortos y el manejo de grandes muestras que exigen agilizar los procesos de investigación y acercar la naturaleza diversa de los datos y de sus formas expresivas. Dichos condicionantes interrogan las prácticas tradicionales de procesamiento de información donde la capacidad de relacionamiento de datos es muy limitada y con ello la potencia interpretativa corre el riesgo de ser subvalorada.

Sin embargo, como lo muestra el recorrido realizado, muchas herramientas y prácticas que aparecieron, hace ya más de una década no tienen aún un uso generalizado en escenarios específicos es el caso de la UPB, donde, por ejemplo, rutinas básicas de la investigación como la búsquedas y organización de información no están mediadas por el uso de gestores bibliográficos a pensar de que la institución los tiene a disposición e incluso ofrece formación para su utilización.

Así, la apropiación de las TIC en el escenario investigativo se abre campo entre la tradición y la experimentación con nuevas opciones, que para el caso de Iberoamérica, puede afirmarse que se han recorrido tímidamente pero no por ello menos reflexivo el trayecto. Entender el panorama de las transformaciones de las prácticas investigativas mediadas por TIC deberá alentar el compromiso frente a la divulgación de usos innovadores en las ciencias sociales y su incidencia en la transformación metodológica y epistemológica, asuntos de los que apenas se están dimensionando su impacto en la investigación.

\section{Referencias}

Alcock, J., Iphofen, R. (2007). Computer-assisted software transcription of qualitative interviews. Nurse Researcher, 15(1), 16-26. doi: 10.7748/nr2007.10.15.1.16.c6051

Amezcua, M., Gálvez, A. (2002). Los modos de análisis en investigación cualitativa en salud: perspectiva crítica y reflexiones en voz alta. Revista Española de salud pública, 76(5), 423-436. Recuperado de: http://www.redalyc.org/pdf/170/17076505.pdf

Aparaschivei, P. A. (2011). The Use of New Media in Electoral Campaigns: Analysis on the Use of Blogs, Facebook, Twitter and YouTube in the 2009 Romanian Presidential Campaign. Journal of Media Research, 4(2), 39-60. Recuperado de: http://connection.ebscohost.com/c/articles/62035692/use-new-media-electoral-

Citación artículo: Álvarez Cadavid, G., Giraldo Ramírez, M., Navarro Plazas, C. (2017). Uso de TIC en investigación cualitativa: discusión y tendencias en la literatura. Revista Katharsis, N 23, enerojunio 2017, pp.218-235, Disponible en http://revistas.iue.edu.co/index.php/katharsis 
campaigns-analysis-use-blogs-facebook-twitter-youtube-2009-romanianpresidential-campaign

Armony, V. (1997). El análisis de datos cualitativos en ciencias sociales: nuevos enfoques y herramientas. Revista de Investigaciones Folklóricas 12, 9-16. Recuperado de: https://www.google.com.co/url? sa=t\&rct=j\&q=\&esrc=s\&source=web\&cd=1\&cad= rja\&uact=8\&ved=0ahUKEwiAlb6IzrjRAhXLg5AKHQaMAvgQFggfMAA\&url=ht tp\%3A\%2F\%2Ffiles.mytis.webnode.cl\%2F200000013-

3e1273f0e1\%2FArmony\%2C\%2520V.\%253B\%2520\%2520El\%2520an\%25C3\%2

5A1lisis $\% 2520$ de $\% 2520$ datos $\% 2520$ cualitativos $\% 2520 \mathrm{en} \% 2520$ ciencias $\% 2520$ soci ales $\% 253 \mathrm{~B} \% 2520$ nuevos $\% 2520$ enfoques $\% 2520 \mathrm{y} \% 2520$ herramientas.pdf\&usg=AF QjCNHnd2E4Pd-TKvN1kYmMBFueofM96Q\&bvm=bv.143423383,d.Y2I

Arriazu, R. (2007). ¿Nuevos medios o nuevas formas de indagación?: Una propuesta metodológica para la investigación social on-line a través del foro de discusión. Forum Qualitative Sozialforschung / Forum: Qualitative Social Research, 8(3), 1-17 Recuperado de:

http://www.qualitativeresearch.net/index.php/fqs/article/viewFile/275/606

Austin, J., Hickey, A. (2009). Working visually in community identity ethnography. International Journal of the Humanities, 7(4), 1-14. Recuperado de: https://eprints.usq.edu.au/5800/1/Hickey_Austin_IJH_v7n4_PV.pdf

Bourdon, S. (2002). The Integration of Qualitative Data Analysis Software in Research Strategies: Resistances and Possibilities. Forum Qualitative Sozialforschung / Forum: Qualitative Social Research, 3(2), 1-12. Recuperado de: http://www.qualitative-research.net/index.php/fqs/article/view/850/1847.

Bartolomé, A. (2008). Vídeo digital y educación: Síntesis.

Brown, D. (2002). Going digital and staying qualitative: Some alternative strategies for digitizing the qualitative research process. Forum Qualitative Sozialforschung/Forum: Qualitative Social Research, 3(2), 1-17. Disponible en http://www.qualitative-research.net/index.php/fqs/article/view/851/1849.

Campos, M., Godoy, A. (2008). Computer-aided qualitative data analysis: emerging questions. RAM. Revista de Administração Mackenzie, 9(4), 75-98. Recuperado de: http://www.scielo.br/pdf/ram/v9n4/v9n4a06

Carvajal, D. (2002). Las herramientas de la artesana. Aspectos críticos en la enseñanza y aprendizaje de los CAQDAS. Forum Qualitative Sozialforschung / Forum: Qualitative Social Research, 3.

Cisneros Puebla, C. A. (2003). Análisis cualitativo asistido por computadora. Sociologias, (9), 288-313, Disponible en https://dx.doi.org/10.1590/S1517-45222003000100010.

Cisneros Puebla, C. A., Davidson, J. (2012). Qualitative Computing and Qualitative Research: Addressing the Challenges of Technology and Globalization. Forum Qualitative Sozialforschung / Forum: Qualitative Social Research, 13(2), 1-11. Disponible en http://www.qualitativeresearch.net/index.php/fqs/article/view/1853/3391

Cisneros Puebla, C., Figaredo, D., Faux, R., Kölbl, C., Packer, M. (2006). Editorial: De epistemologías y periferias en investigación cualitativa. Forum Qualitative Sozialforschung / Forum: Qualitative Social Research, 7(4). 1-6. Disponible en http://www.qualitative-research.net/index.php/fqs/article/viewFile/158/350

Citación artículo: Álvarez Cadavid, G., Giraldo Ramírez, M., Navarro Plazas, C. (2017). Uso de TIC en investigación cualitativa: discusión y tendencias en la literatura. Revista Katharsis, N 23, enerojunio 2017, pp.218-235, Disponible en http://revistas.iue.edu.co/index.php/katharsis 
Coffey, A., Holbrook, B., Atkinson, P. (1996). Qualitative data analysis: Technologies and representations. Sociological Research Online, 1(1), Recuperado de: http://www.socresonline.org.uk/1/1/4.html

Colás, P., De Pablos, J. (2012). Aplicaciones de las tecnologías de la información y la comunicación en la investigación cualitativa. Revista Española de Pedagogía, 70(251), 77-92. Recuperado de: https://dialnet.unirioja.es/servlet/articulo?codigo $=3803557$

Cope, D. (2014). Computer-Assisted Qualitative Data Analysis Software. Oncology Nursing Forum, 41(3), 322-323, Recuperado de: http://web.a.ebscohost.com.consultaremota.upb.edu.co/ehost/detail/detail?vid=10\&s id=95154fe 5 -c6c 9 -43ea- 8957 595686f5f147\%40sessionmgr4010\&hid=4206\&bdata=Jmxhbmc9ZXMmc210ZT1la G9zdC1saXZl\#AN=95758572\&db=a9h. doi:10.1188/14.ONF.322-323

Corredor-Álvarez, F., Íñiguez-Rueda, L. (2016). La foto-provocación como método. Su aplicación en un estudio de la autonomía en personas con diagnóstico de Trastorno Mental Severo. Empiria. Revista De MetodologíA De Ciencias Sociales, O(35), 175204, doi:http://dx.doi.org/10.5944/empiria.35.2016.17173

Chernobilsky, L. (2006). El uso de la computadora como auxiliar en el análisis de datos cualitativos. Estrategias de Investigación Cualitativa. Barcelona: Gedisa.

Crowley, C., Harré, R., Tagg, C. (2002). Qualitative research and computing: methodological issues and practices in using QSR NVivo and NUD*IST. International Journal of Social Research Methodology, 5(3), 193-197. doi:10.1080/13645570210146258.

De Bakker, F., Hellsten, I. (2013). Capturing Online Presence: Hyperlinks and Semantic Networks in Activist Group Websites on Corporate Social Responsibility. Journal of Business Ethics, 118(4), 807-823. http://doi.org/10.1007/s 10551-013-1962-1

Estalella, A., Ardévol, E. (2011). E-research: desafíos y oportunidades para las ciencias sociales. Convergencia, 18(55), 87-111. Recuperado de: http://cccd.es/wp/wpcontent/uploads/2012/05/10515210004.pdf

Fellinghauer, B., Roth, A., Bugari, K., Reinhardt, J. (2011). Construct Validity, Test-Retest Reliability, and Internal Consistency of the Photo Elicitation Semantic Differential Scale (PESD) in Disability Studies. Journal of Developmental \& Physical Disabilities, 23(3), 257-265. Disponible en http://doi.org/10.1007/s10882-010-9224y

Fielding, N., Lee, R. (1996). Qualitative data analysis: representations of a technology: a comment on Coffey, Holbrook and Atkinson.

Fielding, N. (2012). The Diverse Worlds and Research Practices of Qualitative Software. Forum Qualitative Sozialforschung / Forum: Qualitative Social Research, 13(2), 118. Disponible en http://nbn-resolving.de/urn:nbn:de:0114-fqs1202124

Fielding, N., Cisneros, C. (2009). CAQDAS-GIS Convergence:Toward a New Integrated Mixed Method Research Practice? Journal of mixed methods research, 3(4), 349370, doi/abs/10.1177/1558689809344973

Gibbs, G. (2013). Using Software in Qualitative Analysis. En U. Flik (Ed), The SAGE Handbook of Qualitative Data Analysis. (pp. 277-296). NY: Editorial Sage.

Giglietto, F., Rossi, L., Bennato, D. (January 01, 2012). The Open Laboratory: Limits and Possibilities of Using Facebook, Twitter, and YouTube as a Research Data

Citación artículo: Álvarez Cadavid, G., Giraldo Ramírez, M., Navarro Plazas, C. (2017). Uso de TIC en investigación cualitativa: discusión y tendencias en la literatura. Revista Katharsis, N 23, enerojunio 2017, pp.218-235, Disponible en http://revistas.iue.edu.co/index.php/katharsis 
Source.Journal of Technology in Human Services, 30(3-4), 145-159. doi:10.1080/15228835.2012.743797.

Giraldo-Ramírez., M., Álvarez-Cadavid G., Navarro., C., (2016). Panorama de la producción científica sobre usos de TIC en investigación cualitativa. Inedito:Trabajo en revisión para publicación.

Hill, L. (2014). "Some of it I haven"t told anybody else': Using photo elicitation to explore the experiences of secondary school education from the perspective of young people with a diagnosis of Autistic Spectrum Disorder. Educational \& Child Psychology, 31(1), 79-89. Recuperado de:

http://web.a.ebscohost.com.consultaremota.upb.edu.co/ehost/pdfviewer/pdfviewer? vid=10\&sid=9cdc714b-b769-4aea-b0d8-

$86346672 \mathrm{a} 2 \mathrm{f} 2 \% 40$ sessionmgr4010\&hid $=4212$

Kelle, U. (1997). Theory building in qualitative research and computer programs for the management of textual data. DOI: $10.5153 /$ sro.86

Kosik, K. S. (2008). The Wikification of Knowledge. Nieman Reports. 62(4), 42-44. Disponible http://web.b.ebscohost.com.consultaremota.upb.edu.co/ehost/pdfviewer/pdfviewer? sid=f45173db-d5d1-462f-861f-288e4f61ee26\%40sessionmgr105\&vid=4\&hid=116

Lang, P., Gouveia, F., Leta, J. (2013). Cooperation in Health: Mapping Collaborative Networks on the Web. PLoS ONE, 8(8): e71415, doi:10.1371/journal.pone.0071415

Laukkanen, M. (2012). Comparative Causal Mapping and CMAP3 Software in Qualitative Studies. Forum Qualitative Sozialforschung / Forum: Qualitative Social Research, 13(2), $\quad 1$ - 25. Disponible en http://www.qualitativeresearch.net/index.php/fqs/article/view/1846/3372

Lee, R. M., Esterhuizen, L. (2000). Computer software and qualitative analysis: Trends, issues, and responses. International Journal of Social Research Methodology, 3(3), 231-243. Recuperado de:

http://web.a.ebscohost.com.consultaremota.upb.edu.co/ehost/pdfviewer/pdfviewer? vid=16\&sid=9cdc714b-b769-4aea-b0d8$86346672 \mathrm{a} 2 \mathrm{f} 2 \% 40$ sessionmgr4010\&hid $=4212$

Liu, E., Shih, R, Tsai, Y. (2011). Hyperlink network analysis of the educational blog. British Journal of Educational Technology, 42(2), 25-29. doi:10.1111/j.14678535.2010.01150.x

López M., Valdivia A., Fernández R. (2016). Producciones narrativo-visuales y voz de los y las estudiantes: indagación sobre los significados de participación en niños, niñas y jóvenes en escuelas municipales en Chile. Forum Qualitative Sozialforschung / Forum: Qualitative Social Research, 17(1), 1-28, Disponible en http://www.qualitative-research.net/index.php/fqs/article/view/2244/3911

MacMillan, K. (2005). More than just coding? Evaluating CAQDAS in a discourse analysis of news texts. Forum Qualitative Sozialforschung / Forum: Qualitative Social Research, 6(3), 1-18. Disponible en http://www.qualitativeresearch.net/index.php/fqs/article/view/28/59

MacMillan, K., Koenig, T. (2004). The Wow Factor Preconceptions and Expectations for Data Analysis Software in Qualitative Research. Social science computer review, 22(2), 179-186. doi: 10.1177/0894439303262625

Citación artículo: Álvarez Cadavid, G., Giraldo Ramírez, M., Navarro Plazas, C. (2017). Uso de TIC en investigación cualitativa: discusión y tendencias en la literatura. Revista Katharsis, N 23, enerojunio 2017, pp.218-235, Disponible en http://revistas.iue.edu.co/index.php/katharsis 
Meyer, E., Dutton, W. (2009). Top-Down e-Infrastructure Meets Bottom-Up Research Innovation: The Social Shaping of e-Research. Prometheus, 27(3), 239-250. doi:10.1080/08109020903127810

O’brien, M., Vargas- Atkins, T., Umoquit, M., Tso, P. (2012). Cultural-historical activity theory and "the visual"in research: exploring the ontological consequences of the use of visual methods. International Journal of Research \& Method in Education, 35(3), 251-268. http://dx.doi.org/10.1080/1743727X.2012.717433

Pardo, N., Pardo, M., D'Angelo, C. (2012). Discurso en la Web: pobreza en YouTube. Bogotá: Universidad Nacional de Colombia.

Parmeggiani, P. (2009). Going digital: Using new technologies in visual sociology. Visual Studies, 24(1), 71-81. doi: 10.1080/14725860902732991

Pérez Da Silva, J. et al. (2013). Las empresas en Facebook y Twitter. Situación actual y estrategias comunicativas. Revista Latina de Comunicación Social, 68, 676695. Recuperado de: http://www.revistalatinacs.org/068/paper/996_Bilbao/RLCS_paper996.pdfdoi: 10.4185/RLCS-2013-996.

Roberts, K., Wilson, R. (2002). ICT and the research process: Issues around the compatibility of technology with qualitative data analysis. Forum Qualitative Sozialforschung/Forum: Qualitative Social Research, 3(2), Art, 23. Recuperado de: http://www.qualitative-research.net/index.php/fqs/article/viewArticle/862/1872\#gcit

Rodik, P., Primorac, J. (2015). To Use or Not to Use: Computer-Assisted Qualitative Data Analysis Software Usage among Early-Career Sociologists in Croatia. Forum: Qualitative Social Research, 16(1), 1-21 http://www.qualitativeresearch.net/index.php/fqs/article/view/2221

Su, B., Bonk, C. J., Magjuka, R. J., Liu, X., Lee, S. (2005). The Importance of Interaction in web-Based Education: A Program-level Case Study of Online MBA Courses. Journal of Interactive Online Learning, 4(1), 1-19. Recuperado de: http://www.ncolr.org/jiol/issues/pdf/4.1.1.pdf

Tapscott, D., Williams, A. D. (2007). Wikinomics: La nueva economía de las multitudes inteligentes. Barcelona: Paidós.

Tonge, J., Moore, S., Ryan, M., Beckley, L. (2013). Using Photo-Elicitation to Explore Place Attachment in a Remote Setting. Electronic Journal of Business Research Methods, 11(1), 41-50. Recuperado de:

https://www.google.com.co/url?sa=t\&rct=j\&q=\&esrc=s\&source=web\&cd=1\&cad= rja\&uact=8\&ved=0ahUKEwjvuPuXm7vRAhWD5SYKHTSdAEoQFggaMAA\&url $=$ http $\% 3 \mathrm{~A} \% 2 \mathrm{~F} \% 2 \mathrm{Fwww}$. jbrm.com $\% 2$ Fissue $\% 2$ Fdownload.html\%3FidArticle $\% 3$ D290\&usg=AFQjCNHMXf6036pOLISJoOoxXtznr4hWyg\&sig2=RJPBFSVGyDD 2wuM6HBacVw

Valles, M. (2001). Ventajas y desafíos del uso de programas informáticos (eg ATLAS. ti y MAXqda) en el análisis cualitativo. Una reflexión metodológica desde la grounded theory y el contexto de la investigación social española. Recuperado de: http://public.centrodeestudiosandaluces.es/pdfs/S200105.pdf.

Valles, M. (2005). Metodología y tecnología cualitativas: actualización de un debate, desde la mirada más atenta en la obra de Barney G. Glaser. Empiria: Revista de metodología de ciencias sociales(9), 145-168. Recuperado de: http://www.redalyc.org/articulo.oa?id=297125218006

Citación artículo: Álvarez Cadavid, G., Giraldo Ramírez, M., Navarro Plazas, C. (2017). Uso de TIC en investigación cualitativa: discusión y tendencias en la literatura. Revista Katharsis, N 23, enerojunio 2017, pp.218-235, Disponible en http://revistas.iue.edu.co/index.php/katharsis 
Wells, F., Ritchie, D., McPherson, A. (2013). "It is life threatening but I don"t mind'. A qualitative study using photo elicitation interviews to explore adolescents' experiences of renal replacement therapies. Child: Care, Health \& Development, 39(4), 602-612, http://doi.org/10.1111/j.1365-2214.2012.01399.x

Zelger, J., Oberprantacher, A. (2002). Processing of verbal data and knowledge representation by GABEK®-WinRelan®. Forum Qualitative Sozialforschung/Forum: Qualitative Social Research, 3(2), 1-35. Recuperado de: http://www.qualitative-research.net/index.php/fqs/article/view/866/1883

Citación artículo: Álvarez Cadavid, G., Giraldo Ramírez, M., Navarro Plazas, C. (2017). Uso de TIC en investigación cualitativa: discusión y tendencias en la literatura. Revista Katharsis, N 23, enerojunio 2017, pp.218-235, Disponible en http://revistas.iue.edu.co/index.php/katharsis 